\title{
Analytical description of spectral hole-burning effects in active semiconductors
}

\author{
Salvador Balle \\ Departamento de Física Interdisciplinar, Instituto Mediterráneo de Estudios Avanzados \\ (Consejo Superior de Investigaciones Científicas-Universitat de les Illes Balears), E-07071 Palma de Mallorca, Spain
}

Received July 3, 2002

An analytical description of the effects of spectral hole burning on the optical properties of active semiconductor materials is developed for fields that are slow compared to intraband relaxation times. Nonlinear gain compression and four-wave mixing effects are discussed. (C) 2002 Optical Society of America

OCIS codes: $250.5980,140.3280$.

Active semiconductor devices exhibit strong optical nonlinearities because of their sensitivity to light fields. The optical power modifies both the total carrier density and its distribution over the energy bands, thereby changing the gain and the refractive index in the active region. These ultrafast optical nonlinearities may allow for efficient all-optical signal processing in wavelength-division multiplexed networks; actually, all-optical wavelength conversion of the signal, data-format translation, and add-drop functionalities have been demonstrated by use of semiconductor optical amplifiers ${ }^{1,2}$ by means of cross-gain modulation, ${ }^{3}$ cross-phase modulation, ${ }^{4}$ or four-wave mixing $^{5}$ (FWM).

The modeling of these processes requires a proper description of the coupling between the active semiconductor material and the optical field, and spectral hole burning (SHB) should be considered because relatively strong optical fields are commonplace, especially in pulsed operation. A rigorous approach to the interaction between a semiconductor material and an optical field would be based on the microscopic semiconductor Bloch equations, ${ }^{6}$ which incorporate the full dynamics of each electronic state including many-body effects. Such a model offers a satisfactory description of the material properties, although it is highly complex, thus requiring intensive numerical computation, which renders it impractical for device analysis and modeling. For these reasons, the densitymatrix formalism is often used in calculations of the saturated gain and refractive-index spectra from the electronic structure of the semiconductor, ${ }^{7-10}$ but it is often assumed that the field consists of a saturating pump plus a weak probe, and a perturbative expansion in the probe amplitude is invoked to describe SHB. ${ }^{7-9,11}$ In this framework, an important many-body effect (bandgap renormalization) can be phenomenologically included by means of a suitable parameterization of the bandgap energy on the total carrier density. Another important many-body effect (Coulomb enhancement of the gain) has proved more elusive, and nowadays no such parameterization is available.

In this Letter I develop an approximation to the optical response of the medium that describes its frequency and its carrier-density dependence while taking into account SHB for arbitrary field amplitudes. This approximation is based on the field's evolving slowly compared to intraband relaxation, whose characteristic time is $T \sim 0.1 \mathrm{ps}$. Hence it is valid for analyzing dynamics on longer time scales, of the order of picoseconds; thus optical fields with a spectral width of as much as $1 \mathrm{THz}$, still of significance for the present level of the technology, can be considered.

In the presence of an optical field $E=$ $A(t) \exp (-i \Omega t)+$ c.c., whose amplitude $A(t)$ evolves slowly compared to optical frequency $\Omega$, the intraband electron and hole distributions are given, in the rotating-wave approximation, by ${ }^{12}$

$$
\begin{aligned}
& d_{t} n_{k}=-\frac{n_{k}-\bar{n}_{k}}{\tau_{k, n}}+i g_{k}\left(A p_{k}{ }^{*}-A^{*} p_{k}\right), \\
& d_{t} h_{k}=-\frac{h_{k}-\bar{h}_{k}}{\tau_{k, h}}+i g_{k}\left(A p_{k}{ }^{*}-A^{*} p_{k}\right), \\
& d_{t} p_{k}=-\Gamma_{k} p_{k}-i g_{k}\left(n_{k}+h_{k}-1\right) A,
\end{aligned}
$$

where $p_{k}$ is the electron-hole coherence. $\Gamma_{k}=$ $\gamma_{k}+i\left(\omega_{k}-\Omega\right)$ describes the natural width, $\gamma_{k}=(1 / 2)\left(\tau_{k, n}{ }^{-1}+\tau_{k, h}{ }^{-1}\right)$, and $\omega_{k}$ is the resonant frequency of the electronic transition within the relaxation time approximation, which allows for an analytical treatment although it leads to some distortion of the gain spectrum. ${ }^{6,13}$ In Eqs. (1)-(3), $g_{k}=\mu_{k} / \hbar$ is the coupling strength, $\mu_{k}$ is the transition matrix element, and $\bar{n}_{k}$ and $\bar{h}_{k}$ are the Fermi quasi-equilibrium distributions for electrons and holes, respectively, which are approached at a rate $\tau_{k, n(h)}$. The formal solution for $p_{k}$ reads as

$$
\begin{aligned}
p_{k}= & -i g_{k} \int_{-\infty}^{t} \mathrm{~d} t^{\prime} \exp \left[-\Gamma_{k}\left(t-t^{\prime}\right)\right] A\left(t^{\prime}\right)\left(\bar{n}_{k}+\bar{h}_{k}-1\right. \\
& +i g_{k} \int_{-\infty}^{t^{\prime}} \mathrm{d} t^{\prime \prime}\left\{\exp \left[-\left(t^{\prime}-t^{\prime \prime}\right) \tau_{k, n}\right]\right. \\
& \left.+\exp \left[-\left(t^{\prime}-t^{\prime \prime}\right) \tau_{k, h}\right]\right\} \\
& \left.\times\left[A\left(t^{\prime \prime}\right) p_{k}{ }^{*}\left(t^{\prime \prime}\right)-A^{*}\left(t^{\prime \prime}\right) p_{k}\left(t^{\prime \prime}\right)\right]\right) .
\end{aligned}
$$

Assuming that the field varies little over the slowest intraband relaxation time, the lowest-order [in $\tau_{k, n(h)}$ ] 
Padé approximantal for $p_{k}$ can be found by iteration (see, e.g., Ref. 6, p. 90), and it reads as

$$
p_{k}(t)=g_{k} A(t) \frac{\left(\bar{n}_{k}+\bar{h}_{k}-1\right)\left(\Omega-\omega_{k}-i \gamma_{k}\right)}{\left(\Omega-\omega_{k}\right)^{2}+\gamma_{k}^{2}+\gamma_{k}^{2} \epsilon_{k}|A(t)|^{2}}
$$

where $\epsilon_{k} \equiv 2 g_{k}^{2}\left(\tau_{k, n}+\tau_{k, h}\right) \gamma_{k}{ }^{-1}$. Equation (5) recovers the exact solution of a two-level system interacting with a monochromatic field, ${ }^{11}$ and it remains valid as long as the evolution of the field is much slower than the slowest intraband relaxation rate, i.e., for fields with a spectral width below $1 \mathrm{THz}$, because $\tau_{e} \sim 150 \mathrm{fs}$, $\tau_{h} \sim 50 \mathrm{fs}$. The deviation from the quasi-Fermi distribution appears in $p_{k}$ through the fielddependent broadening of each optical transition as given by $\epsilon_{k}|A|^{2}$. Thus for quasi-monochromatic fields SHB leads to power-induced saturation of the gain and the refractive index. For fields with several optical frequencies, FWM occurs because $p_{k}$ displays frequency components that were originally not present in $A(t)$ and that arise from the beat notes in $|A(t)|^{2}$ even if the total carrier density does not exhibit them.

The total material polarization density induced by the field is $P=B(t) \exp (-i \Omega t)+$ c.c., where $B(t)=$ $V^{-1} \sum_{k} g_{k} p_{k}(t), V$ is the volume of the crystal, and the summation runs over all electronic states. In general, such a summation cannot be performed analytically because (i) the transition energies and dipole moment elements of realistic band structures have a complicated dependence on $k$ and (ii) the Fermi-Dirac distributions for the electrons and holes within the bands do not allow for analytical summation. In what follows, I consider the simplest case of an intrinsic quantum well $(\mathrm{QW})$ in which only one electron and one hole band, both parabolic with effective masses $m_{c(v)}$, interact with the optical field; this situation quite closely corresponds to that of narrow, strongly compressively strained QWs. Moreover, I assume that $\mu_{k}, \tau_{k, n}$, and $\tau_{k, h}$ are constant along the band; hence

$$
\begin{aligned}
B(t)= & A(t)\left[\frac{\Lambda(t)+1}{2 \Lambda(t)} f(t)+\frac{\Lambda(t)-1}{2 \Lambda(t)} f^{*}(t)\right], \\
f(t)= & \frac{g^{2}}{V} \sum_{k} \frac{\bar{n}_{k}+\bar{h}_{k}-1}{\Omega-\omega_{k}+i \gamma \Lambda(t)} \\
= & \frac{m \mu^{2}}{\pi W \hbar^{2}}\left\{\ln \left[1-\frac{b}{u+i \Lambda(t)}\right]\right. \\
& \left.+\sum_{n=0}^{\infty} \frac{1}{[u+i \Lambda(t)]^{n+1}}\left[F_{c}^{(n)}+F_{v}^{(n)}\right]\right\},
\end{aligned}
$$

where $\Lambda(t)=\left[1+\epsilon|A(t)|^{2}\right]^{1 / 2}, W$ is the thickness of the QW, $m=\left(m_{c}{ }^{-1}+m_{v}{ }^{-1}\right)^{-1}$ is the reduced mass of the electron-hole pair, $b$ is the sum of the energy intervals covered by the conduction and valence bands normalized to the transition width, and $u \equiv\left(\hbar \Omega-E_{t}\right) /(\hbar \gamma)$ is the difference between the photon energy and the (renormalized) bandgap energy scaled to the natural width of the transitions. Bandgap renormalization is phenomenologically included as $E_{t}=\hbar \Omega_{0}-s N^{1 / 3}$. Finally, $F_{j}^{(n)}=\int_{0}^{\infty} \mathrm{d} x x^{n}\left\{1+\exp \left[a_{j}\left(x-x_{j}\right)\right]^{1 / 2}\right\}^{-1}$ $(j=c, v)$ denotes the Fermi-Dirac integral of order $n$ for the conduction (valence) band, where $a_{c(v)}=$ $(\hbar \gamma) /\left(K_{B} T\right)\left[m / m_{c(v)}\right], K_{B}$ is Boltzmann's constant, $T$ is the temperature, $x_{c(v)}=\left[m_{c(v)} / m\right]\left[\mathcal{F}_{c(v)} / \hbar \gamma\right]$, and the quasi-Fermi levels $\mathcal{F}_{c(v)}$ are measured from the corresponding band edges and linked to the carrier density through $N=(m \gamma) /(\pi W \hbar) F_{j}{ }^{(0)}$.

In the degenerate limit, $F_{j}^{(n)} \approx x_{j}{ }^{n+1} /(n+1)$; hence

$$
\begin{aligned}
f(t)= & \frac{m \mu^{2}}{\pi W \hbar^{2}}\left\{-2 \ln \left[1-\frac{D}{u+i \Lambda(t)}\right]\right. \\
& \left.+\ln \left[1-\frac{b}{u+i \Lambda(t)}\right]\right\},
\end{aligned}
$$

where $D=(\pi W \hbar) /(m \gamma) N \equiv N / N_{t}$ is the scaled carrier density. In the absence of SHB, $\Lambda(t)=1$, and $f(t)$ coincides with the optical susceptibility of the QW medium $^{14}$; in the saturated case, the optical response is found by direct substitution of Eq. (9) into Eq. (6).

For a field with a single optical frequency, one can approximately determine the gain (but not the refractive-index change) even in the nondegenerate limit by considering that the width of each electronic transition is much less than the thermal energy (typically, $\hbar \gamma \sim 6 \mathrm{meV}$, whereas at room temperature $K_{B} T \sim 25 \mathrm{meV}$ ). In this case, from the imaginary part of $B(t) /\left[\epsilon_{0} A(t)\right]$ in Eq. (6) it is found that

$$
\begin{aligned}
G[\Omega, N] \sim & \frac{m \mu^{2}}{\epsilon_{0} \pi W \hbar^{2}} \frac{1}{\Lambda(t)}\left\{\frac{\pi}{2}+\arctan \left[\frac{u}{\Lambda(t)}\right]\right\} \\
& \times\left[\frac{\exp \left(a_{c} D\right)-1}{\exp \left(a_{c} D\right)-1+\exp \left(a_{c} u\right)}\right. \\
& \left.+\frac{\exp \left(a_{v} D\right)-1}{\exp \left(a_{v} D\right)-1+\exp \left(a_{v} u\right)}-1\right] .
\end{aligned}
$$

Both now and in the degenerate limit it is found that the gain is compressed according to $\Lambda=\left(1+\epsilon|A|^{2}\right)^{1 / 2}$; typically, $\mu \sim 10^{-28} \mathrm{C}$, so the gain-compression coefficient (expressed as the inverse of the photon density) is $\epsilon \sim 10^{-17} \mathrm{~cm}^{3}$, in agreement with the commonly measured values of this parameter. ${ }^{15}$ The same functional dependence for nonlinear gain compression as that which is due to SHB in semiconductor media was previously found by Agrawal. ${ }^{16}$ In the present treatment, however, no separation is made between the linear and the nonlinear parts of the gain, thus allowing a simple analytical approximation to be deduced not only for the nonlinear gain coefficient but also for the frequency dependence of the saturated gain spectrum.

Figure 1 displays the gain as a function of the optical frequency of a quasi-monochromatic field for two different carrier densities in the active region, assuming a temperature of $350 \mathrm{~K}$. Two photon densities are considered, $\epsilon|A|^{2}=0$ (solid curves) and $\epsilon\left|A^{2}\right|=0.1$ (dashed curves). It is clearly shown that, as the carrier density is increased, the gain spectrum widens and blueshifts because of band filling, which is partially compensated for by bandgap renormalization. Moreover, for a constant carrier density the material gain spectrum flattens as the optical power level is increased. The transparency level, determined by the 


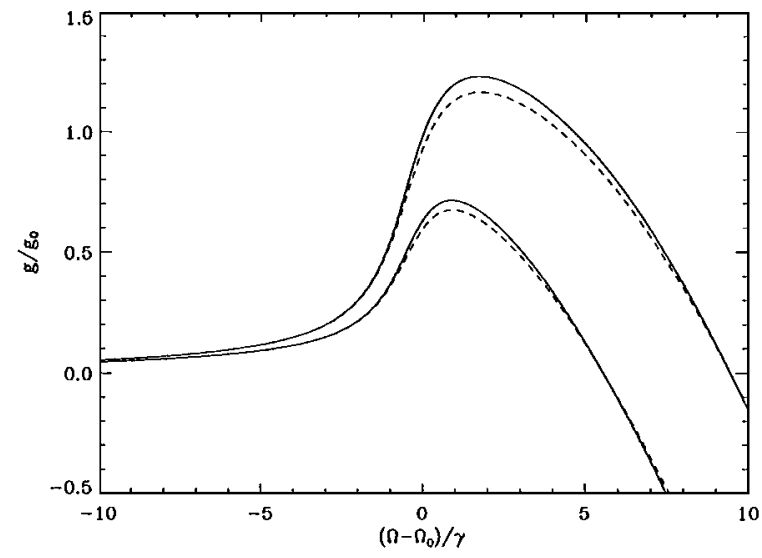

Fig. 1. Gain spectrum at $T=350 \mathrm{~K}$ for two carrier densities, $N=9 N_{t}$ (lower pair of curves) and $N=12 N_{t}$ (upper curves) for photon densities $\epsilon|A|^{2}=0$ (solid curves) and $\epsilon|A|^{2}=0.1$ (dashed curves). Unsaturated transparency, $N=5 N_{t}$.

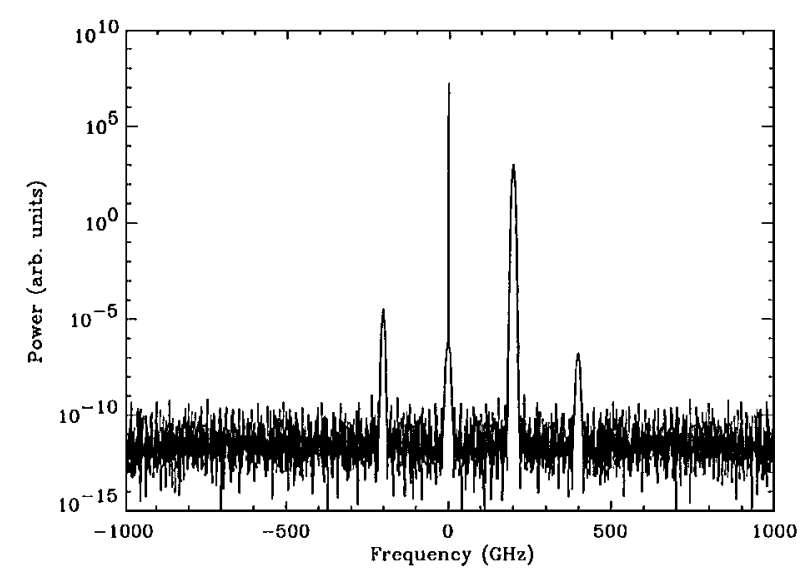

Fig. 2. Spectrum of the material response in the degenerate limit under pulsed conditions (see text for details).

quasi-Fermi level separation of electrons and holes, sets the crossing point for the curves that correspond to various optical powers. For optical frequencies between this level and the renormalized bandgap energy, the gain is depleted as a result of stimulated emission induced by the optical field, whereas for the frequencies above this level the optical field produces a partial bleaching of the absorption.

Figure 2 shows, in the degenerate limit and for $D=3$, the power spectrum of the material response to a cw field of amplitude $E_{\mathrm{cw}}=0.1 \epsilon^{-1 / 2}$ whose frequency corresponds to the maximum of the linear gain superimposed upon a Gaussian optical pulse of $\sim 75$-ps full width at half-maximum, amplitude $E_{\text {pulse }}=0.01 \epsilon^{-1 / 2}$, and detuned $200 \mathrm{GHz}$ to the blue of the cw beam. Clear FWM sidebands are observed that arise solely from intraband dynamics, inasmuch as the carrier density is constant. In a semiconductor optical amplifier, for which the carrier density may have an inhomogeneous distribution and exhibit the beat notes of the various field components, additional FWM contributions may occur.

In summary, I have presented an analytical approximation of the effects of spectral hole burning in active semiconductor media by strong fields that are slow compared to intraband dynamics. The simplest case of a quantum well system with only one electron and one hole band, both parabolic, has been discussed, and the approximation can readily be extended to multiple bands or bulk media. The approximation presented provides a numerically efficient way of describing gain saturation and four-wave mixing in the optical response of a medium while maintaining its dependence on carrier density and wavelength.

I acknowledge many helpful discussions with I. Esquivias and Jorge R. Tredicce. I also acknowledge financial support from Ministerio de Ciencia y Tecnología and the European Commission under projects TIC99-0645 VISTA HP-TRN BFM2000-1108.

\section{References}

1. See, e.g., M. N. Zervas, A. E. Willner, and S. Sasaki, eds., Optical Amplifiers and Their Applications, Vol. 16 of OSA Trends in Optics and Photonics Series (Optical Society of America, Washington, D.C., 1997).

2. G. Guekos, ed., Photonics Devices for Telecommunications (Springer-Verlag, Berlin, 1998).

3. J. M. Wiesenfeld, B. Glance, J. S. Perino, and A. H. Gnauck, IEEE Photon. Technol. Lett. 5, 1300 (1993).

4. T. Durhuus, B. Mikkelsen, C. Joergensen, S. Lykke Danielsen, and K. E. Stubkjaer, J. Lightwave Technol. 14, 942 (1996).

5. D. F. Geraghty, R. B. Lee, M. Verdiell, M. Ziari, A. Mathur, and K. J. Vahala, IEEE J. Sel. Top. Quantum Electron. 3, 1146 (1997).

6. W. W. Chow and S. W. Koch, Semiconductor-Laser Fundamentals. Physics of the Gain Materials (Springer-Verlag, Berlin, 1999).

7. J. Mark and J. Moerk, Appl. Phys. Lett. 61, 2281 (1992).

8. J. Mark and J. Moerk, Proc. SPIE 2399, 146 (1995).

9. A. Uskov, J. Mark, and J. Moerk, IEEE J. Quantum Electron. 30, 1769 (1994).

10. R. Gutiérrez-Castrejón, L. Schares, L. Occhi, and G. Guekos, IEEE J. Quantum Electron. 36, 1476 (2000).

11. S. Haroche and F. Hartmann, Phys. Rev. A 6, 1280 (1972).

12. B. Zhao and A. Yariv, in Semiconductor Lasers I, E. Kapon, ed. (Academic, San Diego, Calif., 1999), Chap. 1, and references therein.

13. P. M. Enders, IEEE J. Quantum Electron. 33, 580 (1997).

14. S. Balle, Phys. Rev. A 57, 1304 (1998).

15. See, e.g., R. Nagarajan and J. E. Bowers, in Semiconductor Lasers I, E. Kapon, ed. (Academic, San Diego, Calif., 1999), Chap. 3, and references therein.

16. G. P. Agrawal, J. Appl. Phys. 63, 1232 (1988). 